\title{
3 Research Square

\section{Genetic variations in methotrexate metabolic pathway genes influence methotrexate responses in rheumatoid arthritis patients in Malaysia}

\author{
Hong Xi Sha \\ Sunway University \\ Kumar Veerapen \\ Broad Institute \\ Sook Khuan Chow \\ Sunway Medical Centre \\ Suk Chyn Gun \\ Hospital Tuanku Ja'afar Seremban \\ Ing Soo Lau \\ Hospital Selayang \\ Renee Lay Hong Lim \\ UCSI University \\ Zaliha Zulkifli \\ Sunway University \\ Yoon Yen Yow \\ Sunway University \\ Suat Cheng Peh \\ Sunway University \\ Jung Shan Hwang ( $\nabla$ hwangjs@sunway.edu.my ) \\ Sunway University
}

\section{Research Article}

Keywords: Methotrexate, rheumatoid arthritis, single nucleotide polymorphism (SNP), ATIC, FPGS, GGH, ITPA, Malaysia

Posted Date: October 18th, 2021

DOI: https://doi.org/10.21203/rs.3.rs-963011/v1

License: (c) (i) This work is licensed under a Creative Commons Attribution 4.0 International License. Read Full License 


\section{Abstract}

Methotrexate (MTX) is the most widely used disease-modifying anti-rheumatic drug (DMARD) for rheumatoid arthritis (RA). Many studies have attempted to understand the genetic risk factors that affect the therapeutic outcomes in RA patients treated with MTX. Unlike other studies that focus on the populations of Caucasians, Indian and east Asian countries, this study investigated the impacts of six single nucleotide polymorphisms (SNPs) that are hypothesized to affect the outcomes of MTX treatment in Malaysian RA patients. A total of 647 RA patients from three ethnicities $\left(\mathrm{N}_{\text {Malay }}=153 ; \mathrm{N}_{\text {Chinese }}=326 ; \mathrm{N}_{\text {Indian }}=168\right)$ who received MTX monotherapy (minimum 15 mg per week) were sampled from three hospitals in Malaysia. SNPs were genotyped in patients using TaqMan real-time PCR assay. Data obtained were statistically analysed for the association between SNPs and MTX efficacy and toxicity. Analysis of all 647 RA patients indicated that none of the SNPs has influence on either MTX efficacy or MTX toxicity according to the Chi-square test and binary logistic regression. However, stratification by self-identified ancestries revealed that two out of six SNPS, ATIC C347G (rs2372536) (OR=0.5478, $95 \% \mathrm{Cl}=0.3396-0.8835, \mathrm{p}=0.01321)$ and $A T I C T 675 \mathrm{C}$ (rs4673993) $(\mathrm{OR}=0.5247,95 \% \mathrm{Cl}=0.3248-0.8478, \mathrm{p}=0.008111)$, were significantly associated with MTX adequate response in RA patients with Malay ancestry $(p<0.05)$. As for the MTX toxicity, no significant association was identified for any SNPs selected in this study. Taken all together, ATIC C347G and ATIC T675C can be further evaluated on their impact in MTX efficacy using larger ancestry-specific cohort, and also incorporating high-order gene-gene and gene-environment interactions.

\section{Introduction}

Rheumatoid arthritis (RA) is an autoimmune disorder which abnormally attacks normal joints and results in inflammation. Most epidemiological studies of RA have been done in Western countries, showing a prevalence of RA in the range of $0.5-1.0 \%$ in the USA and northern European countries ${ }^{1}$. In Malaysia, $0.5 \%$ of the population is affected by RA ${ }^{2}$. It should be noted that there are 3 main ancestries in Malaysia, being 69.8\% Malays, $22.4 \%$ Chinese and $6.8 \%$ Indian as according to the 2021 press release by the Department of Statistics Malaysia ${ }^{3}$. Currently, the most commonly prescribed medication for RA is disease-modifying anti-rheumatoid drugs (DMARDs) including conventional synthetic DMARDs (csDMARDS), targeted synthetic DMARDs (tsDMARDS) and biologic DMARDs (bDMARDs). Other prescribed drugs include non-steroidal anti-inflammatory drugs (NSAIDs) and glucocorticoids ${ }^{4}$. Previous csDMARDs such as anti-malarial drugs (chloroquine and hydroxychloroquine) have been in use since the $1950 \mathrm{~s}^{5}$. Since methotrexate (MTX) was readapted in the late 1980s, it has become the most widely used csDMARD 6 .

MTX is a folate anti-metabolite that suppresses disease activity and reduces joint pain. A low dose (15 mg - 25 $\mathrm{mg}$ ) of MTX per week is prescribed to patients through either subcutaneous or oral administration for at least three months and this drug has been proven to be an effective DMARD for RA ${ }^{7,8}$. However, about 30-50\% of RA patients do not respond to MTX and thus ruling out MTX as a treatment option for these non-responders ${ }^{9-13}$. Moreover, up to $35 \%$ RA patients are forced to discontinue MTX due to the adverse drug effects including stomatitis, gastrointestinal upset, headache or minor central nervous system disturbance, hair loss, ulcers, liver toxicity, pancytopenia and pneumonitis ${ }^{6,9-13}$. Despite these limitations, MTX is still the gold standard for the treatment of RA and in Malaysia, the usage of MTX has increased 6-fold from 1997 to $2007^{14}$. Malaysia Clinical Practice Guidelines on RA recommends that patients are to take either an alternative medication or an increased dosage when MTX therapy shows signs of failure in efficacy or side effects ${ }^{15}$. Hence, anti-RA medicine can become unexpectedly lengthy, costly and ranges from not effective to partially effective in meeting treatment expectation. 
Available literature has converged to hypothesize that the variability in MTX efficacy and toxicity is due to a dysregulation in the MTX pathway (Fig. 1) ${ }^{16-18}$. As multiple enzymes mediate the metabolism of MTX, it is conceivable that the alterations of enzymes' availability and activity have a direct impact on MTX treatment. Our study focused on the potential dysregulation of 4 key enzymes: folylpolyglutamate synthase (FPGS), yglutamyl hydrolase (GGH), aminoimidazole-4-carboxamide ribonucleotide (AICAR) transformylase, and inosine triphosphate pyrophosphatase (ITPA). FPGS is an important enzyme responsible for converting MTX into a range of polyglutamate forms - methotrexate polyglutamate (MTX PG) and is therefore important to allow the retention of MTX bioavailable in the cell. The therapeutic effects of MTX in RA patients rely on its conversion to MTX PG ${ }^{18}$. The conversion of MTX to MTX PG by FPGS can be reversed by $\mathrm{GGH}^{16}$ (Fig. 1). GGH removes the polyglutamates from MTX PG by performing serial trimming on the long chain MTX PG to yield MTX which is able to be exported from the cell. AICAR transformylase (encoded by ATIC gene). AICAR transformylase converts AICAR to formyl-AICAR (FAICAR), playing a role in the de novo purine synthesis (Fig. 1). MTX is able to block AICAR transformylase and hence causes an increase of intracellular level of AICAR. High level of AICAR inhibits the adenosine deaminase (ADA) and AMP deaminase, resulting in accumulation of intracellular adenosines ${ }^{17}$. These adenosines are then transported out of the cell into the extracellular compartment where they bind to adenosine receptors, notably the $\mathrm{A} 2 \mathrm{a}$ and $\mathrm{A} 3$ receptors that activate the adenosine signaling pathway ${ }^{19}$. Active adenosine signaling can lead to multiple anti-inflammatory mechanisms, one of which is the inhibition of NF-kB signaling in fibroblast-like synoviocytes at the synovial joints, thus suppressing the cell proliferation and the secretion of inflammatory factors that causes dysregulated angiogenesis in RA. Other anti-inflammatory mechanisms include the inhibition of proinflammatory activities in neutrophils, macrophages, T-cells and endothelial cells ${ }^{20-22}$. ITPA is also played an important role in the adenosine signaling pathway. The presence of C94A (rs1127354; chr20:3213196) mutation may modulate the ability of ITPA to convert inosine triphosphate (ITP) to inosine monophosphate (IMP) required for de novo purine synthesis which will affect the amount of intracellular adenosines which can be exported for binding to adenosine receptors ${ }^{19}$, leading to multiple anti-inflammatory mechanisms.

Since FPGS, GGH, AICAR and ITPA are known to determine the rate limiting steps in MTX metabolism, the effects of genetic variations such as single nucleotide polymorphisms (SNPs) could modulate the pharmacokinetic properties of these enzymes ${ }^{17,23-26}$. In our study, six SNPs were selected for the genotyping analysis as based on their previously reported association with MTX treatment outcomes. These SNPs include FPGSA1994G (rs10106; chr9:127813796), GGHC452T (rs11545078; chr8:63026205), GGHC401T (rs3758149; chr8:63039169), ATIC C347G (rs2372536; chr2:215325297), ATIC T675C (rs4673993; chr2:215347616) and ITPA C94A (rs1127354; chr20:3213196). In addition, these candidate gene association studies were previously and mainly conducted in the Caucasian population and no similar study has been carried out for Malaysian RA patients. Therefore, the novelty of this study is to reassess the possible association of the SNPs with MTX treatment outcome in a Malaysian population. We hypothesize that specific SNP changes that can alter gene function are able to explain the variability observed in MTX efficacy and toxicity. Thus, this study aimed to genotype six SNPs of the candidate genes (FPGS, GGH, ATIC and ITPA) in MTX metabolic pathway and determine their association with MTX therapeutic outcomes in Malaysian RA patients.

\section{Results}

\section{Characterization of the studied population}


Demographics. This study recruited 647 RA patients from Sunway Medical Centre ( $n=297)$, Hospital Tuanku Ja'afar Seremban ( $n=304)$ and Hospital Selayang $(n=96)$ (Table 1). The number of Chinese RA patients, most of which were recruited from Sunway Medical Centre, was two times higher than Malay or Indian RA patients. Furthermore, female RA patients (88.7\%) outnumbered male RA patients $(11.3 \%)$ in this study. This sex-imbalance is consistent with the current literature which have shown a higher number of female RA patients.

MTX Efficacy and Toxicity. Based on our criteria for categorization for MTX efficacy, we obtained a total of 252 adequate responders (ARs) and 352 inadequate responders (IRs): $58 \%$ of RA patients did not respond well to MTX (Table 1). As for MTX toxicity, we identified 448 non-adverse drug reaction (Non-ADR) and 199 adverse drug reaction (ADR) patients: 1 in 3 RA patients developed at least one type of side effects during the MTX treatment. Among 199 RA patients who experienced ADRs, 43 patients showed severe side effects and their MTX therapy were immediately ceased (Table 1). These 43 patients were excluded from the MTX efficacy analysis but included in the MTX toxicity analysis.

\section{Differences of allelic and genotype frequencies among 3 ethnic groups}

The TaqMan SNP genotyping assay was performed on all study samples for the following SNPs: FPGS A1994G (rs10106), GGHC452T (rs11545078), GGHC401T (rs3758149), ATIC C347G (rs2372536), ATIC T675C (rs4673993), and ITPA C94A (rs1127354). 5\% of the samples were randomly chosen for each SNPs and then verified by Sanger sequencing. The sequencing results confirmed the accuarry of the TaqMan SNP genotyping assay results. The allelic frequencies and genotype counts for each SNPs in Malay, Chinese and Indian RA patients are shown in Table 2. The minor allele frequency (MAF) of all six SNPs, except ITPA C94A (rs1127354), showed significant variation among the RA patients for the three ethnic groups. The MAFs of ITPA C94A (rs1127354) in Malay, Chinese and Indian patients are $0.15,0.16$ and 0.14 , respectively ( $p>0.05$; solid-line box in Table 2). In addition, genotype counts for the six SNPs were compared among the three ethnic groups using chi-square test. The results revealed that except GGHC452T (rs11545078) and ITPA C94A (rs1127354), other four SNPs significantly differ in genotype frequencies among the Malay, Chinese and Indian RA patients (dashed line boxes in Table 2).

\section{Association of six metabolic SNPs with MTX efficacy and toxicity in three ancestry-specific RA patients}

When the association study of SNPs with the MTX treatment was carried out using the entire cohort ( $n=647)$, there was no significant difference between ARs and IRs as well as Non-ADR groups and ADR groups in the allelic association tests. Logistic regression was then performed to test the standard models of disease penetrance (dominant, recessive, additive) for the interaction of six SNPs with MTX efficacy and toxicity in the cohort of 647 RA patients. The forest plot (Fig. 2) for the association between SNPs and the MTX efficacy and toxicity was performed using $\mathrm{R}$ package ggplot $2^{27,28}$ indicated no significant association between the SNPs with either MTX efficacy or MTX toxicity (Supplementary Table S2 and Supplementary Table S3, respectively).

We then stratified the study cohort into three ancestry-specific groups, Malay, Chinese and Indian. The numbers of $\mathrm{AR}$ and IR of the stratified groups with their respective genotypes are presented in Supplementary Table S4. Using these data, significant differences were observed in ATIC C347G (rs2372536) (OR=0.5478, 95\% Cl=0.3396-0.8835, $\mathrm{p}$ $=0.01321)$ and ATIC T675C (rs4673993) (OR=0.5247, 95\%Cl=0.3248-0.8478, $\mathrm{p}=0.008111)$ (Supplementary Table S6). Based on the effect sizes obtained from our analyses, the risk of patients becoming IR was reduced by ATIC C347G (rs2372536) and ATIC T675C (rs4673993) for approximately 55\% and 57\%, respectively. When the 
inheritance models were applied to the ancestry-specific stratification, it could be inferred that: (i) ATIC C347G (rs2372536) was associated with AR in Malay RA patients under dominant and additive models; (ii) the minor allele of ATIC T675C (rs4673993) under three genetic models (dominant, recessive and additive) may predict a higher success rate in MTX treatment among Malay RA patients (Supplementary Table S6). All positive results for the association between SNPs and MTX efficacy are as shown in the forest plot (Fig. 3). All the six SNPs were not significantly associated with the MTX efficacy in either Chinese or Indian RA patients. Furthermore, there was no significant association of all six SNPs with MTX toxicity in the three ancestry-specific groups (Supplementary Table S5 and Supplementary Table S7).

\section{Discussion}

Majority of the available drugs used for the treatment of RA were clinically evaluated in European ancestries, this raises a concern about their efficacy and toxicity in other ancestry groups globally ${ }^{29}$. Asian populations especially those in South East Asia were considerably under-represented in pharmacogenomic and pharmacogenetic studies of RA ${ }^{29.30}$. Hence, the present study evaluated the outcomes of MTX treatment in three major ancestry groups in Malaysia and their association with 6 SNPs from the enzymes involved in MTX metabolism. Comparing with the studies conducted by geographical locations, our study attempted to delineate ancestry specific risk factors that would increase the precision of the proposed association.

The MAF of ATICT675C (rs4673993) recorded in Genome Aggregation Database (gnomAD) and 1000 Genomes is 0.3251 and 0.2855 , respectively ${ }^{31,32}$. In our study, the allele frequency of ATICT675C (rs4673993) for the overall cohort is 0.4 and it is $0.44,0.31$ and 0.54 , respectively, in Malay, Chinese and Indian populations in Malaysia (Table 2). By comparing the allele frequency between our study and the public database, we noticed that our population is carrying a higher allele frequency of ATIC T675C (rs4673993). When comparing allele frequencies by ethnicity within our study cohort, there was a significant difference between Malay, Chinses and Indian for this SNP. Apparently, the allele frequency of ATICT675C (rs4673993) in Indian and Malay subjects were significantly higher than that observed in Chinese subject and in the public databases.

Interestingly, our study suggested that the Malay RA patients with ATIC T675C (rs4673993) have a better treatment outcome upon MTX monotherapy. In other words, this minor allele was associated with an increased remission rate in Malay RA patients following the treatment of MTX. A few studies have also demonstrated the impact of ATIC T675C (rs4673993) on MTX treatment outcome. Prospective studies conducted by Lee et al. (2009) and lannaccone et al. (2010) have shown that ATIC T675C (rs4673993) was significantly associated with low disease activity in RA patients with MTX monotherapy ${ }^{23,33}$. These two studies were conducted in the USA with 120 and 262 RA patients, respectively, as the subsets of Brigham and Women's Hospital Rheumatoid Arthritis Sequential Study (BRASS). Moreover, a meta-analysis performed by Chen (2017) indicated that ATIC T675C (rs4673993) predicts the responsiveness of MTX treatment ${ }^{34}$. The authors combined two studies to yield a total of 698 Caucasians and observed a significant favouritism of ATIC T675C (rs4673993) in RA patients having response to MTX treatment. On the other hand, a retrospective study by Lima et al. (2014) gave a totally different conclusion ${ }^{35}$, whereby more than 4-fold increase in risk of MTX inefficacy was associated with ATIC T675C (rs4673993) in a population of 233 adults ( $\geq 18$ y.o.) of Portuguese Caucasian RA patients. The result discrepancy may be due to different ancestral lineages of RA patients enrolled in the respective studies. In our case, the association of ATICT675C (rs4673993) with the responsiveness to MTX treatment could only be observed in Malay but not in Chinese and Indian RA patients. 
Current RA literature consistently highlights the hypothesis that the anti-inflammatory action of MTX is achieved through the indirect inhibition of AICAR. The ATICT675C (rs4673993) SNP is positioned in the intronic region of ATIC. To our knowledge, there are no functional studies on this particular SNP in ATIC activity. Nevertheless, the intronic SNP either interferes the transcriptional regulation of the coding-enzyme or is in linkage disequilibrium (LD) with another coding SNP ${ }^{23,36-38}$. In the present study, since similar effect size of ATICT675C (rs4673993) and ATIC C347G (rs2372536) was observed, both SNPs can be in LD. Nevertheless, this observation need further validation, since the current sample size is too small to perform a LD test and the lack of a reference panel for ATICT675C (rs4673993) and ATIC C347G (rs2372536) in Malay patients.

Similar to ATIC T675C (rs4673993), the allele frequency of ATIC C347G (rs2372536) in Malay, Chinese and Indian populations is $0.45,0.31$ and 0.54 , respectively; and the allele frequency of ATIC C347G (rs2372536) for the entire study cohort is 0.40 (Table 2). The allele frequency of ATIC C347G (rs2372536) retrieved from gnomAD, GO-ESP and 1000 Genomes are $0.3172,0.2468$ and $0.2778^{31,32,39}$, respectively. Except the Chinese population, the allele frequencies observed for Malay and Indian are higher than the ones retrieved from the public databases.

Our result suggested Malay RA patients with ATIC C347G (rs2372536) having a better response to MTX treatment as compared to Chinese and Indian RA patients. In fact, this is in alignment with the data previously presented by Dervieux et al. (2004) ${ }^{36}$ on a cross-sectional study of 108 RA patients (age $\geq 18$ y.o.) from a local rheumatology clinic in Knoxville, USA ${ }^{36}$. In their study, patients carrying a homozygous GG of ATIC C347G (rs2372536) may have a higher ratio of good response to MTX compared with patients carrying a CC or CG genotype. Moreover, Kurzawski et al. (2016) ${ }^{40}$ studied 422 Caucasian RA patients in Poland who were treated with MTX therapy and found that GG minor genotype significantly exhibited a good response to MTX. However, the lack of association between rs2372536 polymorphism and the clinical response to MTX was also reported in some studies ${ }^{41,42}$. Recently, two meta-analyses were performed to investigate the association between ATIC C347G (rs2372536) and MTX response ${ }^{43,44}$. The first meta-analysis was based on five studies of 1056 RA patients in which 722 were MTX responders and 334 were non-responders. This analysis found the difference of ATIC C347G (rs2372536) between Caucasions (Spain, Slovenia and Netherlands) and Asians (India), being associated with non-responsiveness to MTX treatment in Caucasians but not associated in Asians ${ }^{43}$. The second meta-analysis combined two European (Spain and Netherlands), one East Asian (Japan) and two South Asian (India) studies with 458 MTX responders and 398 non-responders in total ${ }^{44}$. When combining five studies, ATIC C347G (rs2372536) demonstrated a significant association with non-responsiveness of MTX under the dominant and codominant models. Yet, geographical stratification showed that the association of ATIC C347G with MTX response was still observed in Europeans in pre-allele, dominant and codominant models but not in South Asian populations ${ }^{44}$.

Despite all studies above demonstrated a significant association between ATIC variants and MTX efficacy, the results were raher inconsistent. Common factors for inconsistency such as small sample size and insufficient statistical power, study design, medication dosage, grouping criteria, and patient condition could cause limitations in the association study. Moreover, gene-gene interactions within folate and adenosine biosynthesis pathways may complicate the association study between SNPs and MTX treatment outcomes ${ }^{45}$. In fact, RA has complex inheritance patterns and no single genetic variant has a decisive role in MTX efficacy or MTX toxicity in the treatment of RA. By using the MDR (Multifactor Dimensionality Reduction) method, a cohort of 255 RA patients treated with MTX in the USA was evaluated with the efficacy of MTX treatment, and the results showed that $53 \%$ MTX responders was associated with high-order interactions among SNPs in ITPA (C94A), RFC1 (G80A), and ATIC $(\mathrm{C} 347 \mathrm{G})$ genes $^{45}$. Upon excluding the predisposing genotype combinations, a 3.8-fold lower efficacy was 
observed $^{45}$. Later, the same researchers extended their study of gene-gene interactions using ITPA (C94A), RFC1 (G80A), and ATIC (C347G) to another 3 RA cohorts (USA, Dutch and Swedish) ${ }^{46}$. Both USA and Dutch cohorts $(n=435)$ confirmed a predisposing genetic attribute significantly associated with methotrexate response [odds ratio $(\mathrm{OR})=2.9,95 \%$ confidence interval $(\mathrm{Cl}): 1.9-4.2 ; P<0.001]$. Although the association of combined SNPS with MTX responsiveness in the Swedish cohort $(n=530)$ could not be determined, the association was observed after the non-genetic factors, age, sex and anti-citrullinated protein antibody (ACPA) status were included in MDR analysis ${ }^{46}$. Thus, individual variants of ATIC may not play a direct role in MTX efficacy, future studies shall map the ATIC variants to drug response as based on the detection of nonlinear multigene interactions, this may improve the accuracy of predicting the MTX efficacy. In addition, other non-genetic covariates should be considered because the association study between genetic variants and MTX efficacy sometimes seems oversimplified understanding the MTX response in RA.

AICAR transformylase contains two domains which are MGS (methylglyoxal synthetase) like domain and AICAR binding domain ${ }^{47}$. ATIC C347G (rs2372536) causes the substitution of threonine (Thr) with serine (Ser) at position 116. Thr116 lies in the binding pocket of MGS-like domain and is the first residue of a8 helix which likely serves as a $\mathrm{N}$-cap residue stabilizing the helix by interacting with the amide groups from the main chain. We proposed that the side-chain hydroxyl group of Thr116 forms hydrogen bonds with the amide groups of Val117 and Glu118 (green arrowhead in Fig. 4), while its main-chain carboxyl group forms hydrogen bond with the amide group from Glu119 (blue arrowhead in Fig. 4). The methyl group of Thr116 might stabilize the hydrogen bond between Thr116 and the main chain. As Thr116 is substituted with serine, the methyl group can be removed and this results in a more flexible C-N rotation. In other words, Ser116 causes the rearrangement of the protein structure at N-cap and thus, potentially affects the substrate-binding affinity and AICAR transformylase enzyme activity. This explains why the RA patients with the minor allele of ATIC C347G (rs2372536) might have a phenotypic change in response to MTX.

\section{Conclusion}

Present study suggested that ATIC C347G (rs2372536) and ATIC T675C (rs4673993) could influence the response to MTX monotherapy in Malay patients with RA, while the other four SNPs failed to demonstrate their associations with the reduction of disease activity following the MTX monotherapy. ATIC C347G (rs2372536) and ATICT675C (rs4673993) are not the only ancestry-specific SNPs since any variations appear in the genes of MTX metabolic pathway are potentially able to affect the effectiveness of MTX treatment. As more Malay-specific SNPs can be revealed, the prediction of poor response would enable patient to be placed on alternative drugs, while those with predicted good response could proceed with MTX treatment.

As for the future recommendation, ancestry specific signal of ATIC should be validated in a larger replication cohort of a similar ancestry group profile to reduce the Type II error rate of MTX treatment response. Both ATIC C347G (rs2372536) and ATICT675C (rs4673993) warrant an in-depth investigation, especially in the Malay RA patients in Malaysia.

\section{Methods}

\section{Study subjects}


RA patients were recruited at Sunway Medical Centre (Selangor, Malaysia), Hospital Tuanku Ja'afar Seremban (Seremban, Malaysia) and Hospital Selayang (Selangor, Malaysia) from December 2016 to May 2019. The study was performed in accordance with the principles stated in the Declaration of Helsinki. Prior to starting the study, the ethical approval was obtained from the Sunway University Research Ethics Committee (SUNREC 2017/066), the Sunway Medical Centre Independent Ethics Committee (007/2016/ER), and the Medical Research Ethics Committee of Ministry of Health Malaysia (NMRR-17-2901-38245(IIR). RA patients enrolled in this study had fulfilled ACR-EULAR (2010) response criteria and satisfy the inclusion criteria: (i) must be at least 18 years old, (ii) are Malaysian Malay, Chinese or Indian origin, (iii) have been treated with $15 \mathrm{mg}$ MTX or more per week for at least 3 months and (iv) have been followed up 6 months since MTX treatment. The self-declared ancestry of a patient was decided based on both parents being of the same ancestry as the patient. Patients of non-Malaysian origin were excluded from this study. All recruited RA patients were subsequently subjected to two independent association studies on the potential pharmacokinetic impact of SNPs with MTX efficacy and with MTX toxicity.

MTX Efficacy. RA patients were DMARD naive at the time of MTX commencement and they were categorized into adequate responder (AR) and inadequate responder (IR). Adequate responders were interpreted as patients who are in clinical remission or have achieved low disease activity as defined by Disease Activity Score-28 (DAS28CRP) for at least 6 months. On the other hand, inadequate responders have the same RA treatment as adequate responders but failed to achieve clinical remission or low disease activity as defined by DAS28CRP but at present been treated with other DMARDs, mono, duo (excluding MTX and HCQ group) or triple therapy, either csDMARDs or tsDMARDs or bDMARDs. Hence, patients may or may not be on MTX at the point of recruitment.

MTX Toxicity. Patients were categorized into two groups for potential toxicity and side effect: non-adverse drug reaction (Non-ADR) group and adverse drug reaction (ADR) group. The categorization was marked based on whether they have experienced drug intolerance during the MTX treatment. All side effects were recorded from the start of the MTX treatment until the withdrawal due to adverse drug reactions.

\section{Blood sample and clinical data collections}

A total of 647 RA patients were involved in this study after their informed consent was obtained. A total of $5 \mathrm{ml}$ of the whole blood sample was collected in ethylenediaminetetraacetic acid (EDTA) tube from individual patients by venepuncture during patients' regular visits at the hospitals. Besides, clinicopathological and demographic data were also extracted from patients' clinical records and linked to deidentified patient blood samples collected for this study.

\section{TaqMan® SNP Genotyping Assays}

Genomic DNA from patients was isolated from patients' whole blood samples. Briefly, buffy coat was obtained from the blood sample by centrifugation. Genomic DNA was then extracted from the buffy coat by using QIAamp DNA Blood Mini Kit (Qiagen, Germany) according to the manufacturer's instructions. SNP genotyping of FPGS A1994G (rs10106), GGH C452T (rs11545078), GGH C401T (rs3758149), ATIC C347G (rs2372536), ATIC T675C (rs4673993) and ITPA C94A (rs1127354) were performed by using TaqMan® SNP Genotyping Assays (Thermo Fisher, USA) according to manufacturer's instructions. The genotype data of each participant were analyzed using an online software named "Genotyping V4.2" (Thermo Fisher Connect ${ }^{\mathrm{TM}}$ ). A total of $5 \%$ of the samples $(n=33)$ for each respective SNP were randomly selected for PCR amplification (AmpliTaq Gold ${ }^{\text {TM }} 360$ Master Mix, Thermo Fisher Scientific, USA) (Supplementary Table S1) and subsequently for the genotype verification by Sanger 
sequencing (1st BASE Pt Ltd). Sequencing results were curated with SnapGene V4.3.10 (from GSL Biotech; available at https://snapgene.com).

\section{Statistical analysis}

Genotype and allele frequency of all the selected six SNPs were calculated. A chi-square independence test was performed to test the association between SNPs and ethnic groups. Chi-square test and binary logistic regression were performed to investigate the association between SNPs and MTX efficacy and MTX toxicity (PLINK V1.09) ${ }^{48}$. Effect sizes of potential associations were calculated as odds ratio (OR) and 95\% confidence intervals (Cl) as a measure of the association between the categorical variables. A p-value of $<0.05$ was considered to be statistically significant.

\section{Declarations}

\section{Acknowledgement}

We are grateful to Asst. Prof. Elise Robinson and Dr. Raymond Walters for their advice regarding the analysis of genetic data.

\section{Funding}

This study is financially supported by Sunway University (Sunway Internal Grants, INT-2018-SHMS-SIHD-04) and Sunway Medical Centre (SunMed Research Grant, SRC/001/2017/FR).

\section{Authors' contributions}

H.X.S. and Z.Z. carried out the experiments. H.X.S. and K.V. processed the experimental data and performed the statistical analysis. S.K.C., S.C.G. and I.S.L. collected patients' clinical data and blood samples. J.S.H. conceived the original idea and designed the study. H.X.S. wrote the manuscript with the inputs from K.V., R.L.H.L., Y.Y.Y., P.S.C. and J.S.H.

\section{Competing interests}

The authors declare that they have no competing intrerest.

\section{Availability of data and material}

The data underlying this article cannot be shared publicly due to the privacy of individuals that participated in the study. The data will be shared on reasonable request to the corresponding author.

\section{References}

1. Smolen, J. S. et al. Rheumatoid arthritis. Nat. Rev. Dis. Primers, 4, 18001 https://doi.org/10.1038/nrdp.2018.1 (2018).

2. Arthritis Foundation. Types of Arthritis Pain https://www.arthritis.org/health-wellness/healthyliving/managing-pain/understanding-pain/sources-of-arthritis-pain (2021).

3. Department of Statistics Malaysia. Demographic Statistics Second Quarter 2021, Malaysia https://www.dosm.gov.my/v1/index.php? 
r=column/ctwoByCat\&parent_id=115\&menu_id=LOpheU43NWJwRWVSZkIWdzQ4TIhUUT09 (2021).

4. Singh, J. A. et al. 2015 American College of Rheumatology guideline for the treatment of rheumatoid arthritis. Arthritis Rheumatol. 68, 1-26 (2016). https://doi.org/10.1002/art.39480

5. Schrezenmeier, E. \& Dörner, T. Mechanisms of action of hydroxychloroquine and chloroquine: implications for rheumatology. Nat. Rev. Rheumatol, 16, 155-166 https://doi.org/10.1038/s41584-020-0372-x (2020).

6. Weinblatt, M. E. et al. Efficacy of low-dose methotrexate in rheumatoid arthritis. N. Engl. J. Med, 312 (13), 818822 https://doi.org/10.1056/NEJM198503283121303 (1985).

7. Visser, K. \& van der Heijde, D. Optimal dosage and route of administration of methotrexate in rheumatoid arthritis: a systematic review of the literature. Ann. Rheum. Dis, 68 (7), 1094-1099

https://doi.org/10.1136/ard.2008.092668 (2009).

8. Molina, J. T. et al. Recommendations for the use of methotrexate in rheumatoid arthritis: up and down scaling of the dose and administration routes. Reumatol. Clin, 11 (1), 3-8

https://doi.org/10.1016/j.reuma.2014.02.012 (2015).

9. Kinder, A. J. et al. The treatment of inflammatory arthritis with methotrexate in clinical practice: treatment duration and incidence of adverse drug reactions., 44 (1), 61-66 https://doi.org/10.1093/rheumatology/keh512 (2005).

10. Aletaha, D. \& Smolen, J. S. The rheumatoid arthritis patient in the clinic: comparing more than 1,300 consecutive DMARD courses., 41 (12), 1367-1374 https://doi.org/10.1093/rheumatology/41.12.1367 (2002).

11. Maetzel, A. et al. Meta-analysis of treatment termination rates among rheumatoid arthritis patients receiving disease-modifying anti-rheumatic drugs., 39 (9), 975-981 https://doi.org/10.1093/rheumatology/39.9.975 (2000).

12. Albrecht, K. \& Müller-Ladner, U. Side effects and management of side effects of methotrexate in rheumatoid arthritis. Clin. Exp. Rheumatol, 28 (Suppl.61), S95-S101 (2010).

13. Alarcón, G. S. Early rheumatoid arthritis: combination therapy of doxycycline plus methotrexate versus methotrexate monotherapy. Nat. Clin. Pract. Rheumatol, 2, 296-297 https://doi.org/10.1038/ncprheum0195 (2006).

14. Sulaiman, W., Toib, A., Chandrashekhar, G. \& Arshad, A. The trends of DMARDS prescribed in rheumatoid arthritis patients in Malaysia. Oman. Med. J, 24 (4), 260-263 https://doi.org/10.5001/omj.2009.53 (2009).

15. Ministry of Health, Malaysian Society of Rheumatology, \& Academy of Medicine Malaysia. Clinical Practice Guidelines 2019: Management of Rheumatoid Arthritis.

https://www.moh.gov.my/moh/resources/Penerbitan/CPG/2)_CPG_Management_of_Rheumatoid_Arthritis.pdf (9 Oct 2020, date last accessed).

16. Tian, H. \& Cronstein, B. N. Understanding the mechanisms of action of methotrexate: implications for the treatment of rheumatoid arthritis. Bull NYU Hosp. Jt. Dis, 65 (3), 168-173 (2007).

17. Wessels, J. A. M., Huizinga, T. W. J. \& Guchelaar, H. J. Recent insights in the pharmacological actions of methotrexate in the treatment of rheumatoid arthritis., 47 (3), 249-255

https://doi.org/10.1093/rheumatology/kem279 (2008).

18. Kremer, J. M. Toward a better understanding of methotrexate. Arthritis Rheum, 50 (5), 1370-1382 https://doi.org/10.1002/art.20278 (2004).

19. Padovan, M. et al. Adenosine and adenosine receptors in rheumatoid arthritis. Int. J. Clin. Rheumatol, 8 (1), 13-25 https://doi.org/10.2217/ijr.12.76 (2013).

Page $10 / 20$ 
20. Cronstein, B. N. \& Aune, T. M. Methotrexate and its mechanisms of action in inflammatory arthritis. Nat. Rev. Rheumatol, 16 (3), 145-154 https://doi.org/10.1038/s41584-020-0373-9 (2020).

21. Haskó, G. \& Cronstein, B. N. Regulation of inflammation by adenosine. Front. Immunol, 4, 85 https://doi.org/10.3389/fimmu.2013.00085 (2013).

22. Cronstein, B. N., Naime, D. \& Ostad, E. The antiinflammatory mechanism of methotrexate. Increased adenosine release at inflamed sites diminishes leukocyte accumulation in an in vivo model of inflammation. J. Clin. Invest, 92 (6), 2675-2682 https://doi.org/10.1172/JCl116884 (1993).

23. Lee, Y. C. et al. Investigation of candidate polymorphisms and disease activity in rheumatoid arthritis patients on methotrexate., 48 (6), 613-617 https://doi.org/10.1093/rheumatology/ken513 (2009).

24. Lima, A., Bernardes, M., Azevedo, R., Medeiros, R. \& Seabra, V. Pharmacogenomics of methotrexate membrane transport pathway: can clinical response to methotrexate in rheumatoid arthritis be predicted? Int. J. Mol. Sci, 16 (6), 13760-13780 https://doi.org/10.3390/ijms160613760 (2015).

25. Owen, S. A. et al. Genetic polymorphisms in key methotrexate pathway genes are associated with response to treatment in rheumatoid arthritis patients. Pharmacogenomics J, 13 (3), 227-234 https://doi.org/10.1038/tpj.2012.7 (2013).

26. Yanagimachi, M. et al. Influence of polymorphisms within the methotrexate pathway genes on the toxicity and efficacy of methotrexate in patients with juvenile idiopathic arthritis. Br. J. Clin.. Pharmacol, 71 (2), 237-243 https://doi.org/10.1111/j.1365-2125.2010.03814.x (2011).

27. R Core Team. R: A language and environment for statistical computing (2013). http://www.R-project.org

28. Wickham, H. ggplot2: elegant graphics for data analysis (Springer, 2016). https://ggplot2.tidyverse.org

29. Sivadas, A., Salleh, M. Z., Teh, L. K. \& Scaria, V. Genetic epidemiology of pharmacogenetic variants in South East Asian Malays using whole-genome sequences. Pharmacogenomics J.s17(5), 461-470(2017). https://doi.org/10.1038/tpj.2016.39

30. The, H. U. G. O. et al. Mapping human genetic diversity in Asia., 326 (5959), 1541-1545 https://doi.org/10.1126/science.1177074 (2009).

31. Karczewski, K. J. et al. The mutational constraint spectrum quantified from variation in 141,456 humans. Nature, 581 (7809), 434-443 https://doi.org/10.1038/s41586-020-2308-7 (2020).

32. 1000 Genomes Project. Consortium A global reference for human genetic variation. Nature, 526 (7571), 68-74 https://doi.org/10.1038/nature15393 (2015).

33. lannaccone, C. K. et al. Using genetic and clinical data to understand response to disease-modifying antirheumatic drug therapy: data from the Brigham and Women's Hospital Rheumatoid Arthritis Sequential Study., 50 (1), 40-46 https://doi.org/10.1093/rheumatology/keq263 (2011).

34. Chen, Y., Zou, K., Sun, J., Yang, Y. \& Liu, G. Are gene polymorphisms related to treatment outcomes of methotrexate in patients with rheumatoid arthritis? A systematic review and meta-analysis., 18 (2), 175-195 https://doi.org/10.2217/pgs-2016-0158 (2017).

35. Lima, A. et al. Prediction of methotrexate clinical response in Portuguese rheumatoid arthritis patients: implication of MTHFR rs1801133 and ATIC rs4673993 polymorphisms. Biomed. Res. Int. 2014, 368681 (2014). https://doi.org/10.1155/2014/368681

36. Dervieux, T. et al. Polyglutamation of methotrexate with common polymorphisms in reduced folate carrier, aminoimidazole carboxamide ribonucleotide transformylase, and thymidylate synthase are associated with 
methotrexate effects in rheumatoid arthritis. Arthritis Rheum, 50 (9), 2766-2774

https://doi.org/10.1002/art.20460 (2004).

37. Dervieux, T. et al. Pharmacogenetic and metabolite measurements are associated with clinical status in patients with rheumatoid arthritis treated with methotrexate: results of a multicentred cross sectional observational study. Ann. Rheum. Dis, 64 (8), 1180-1185 https://doi.org/10.1136/ard.2004.033399 (2005).

38. Dervieux, T. Methotrexate pharmacogenomics in rheumatoid arthritis: introducing false-positive report probability., 48 (6), 597-598 https://doi.org/10.1093/rheumatology/kep060 (2009).

39. University of Washington. NHLBI Exome Sequencing Project (ESP), Exome Variant Server. http://evs.gs.washington.edu/EVS/ (9 Oct 2020, date last accessed).

40. Kurzawski, M. et al. ATIC missense variant affects response to methotrexate treatment in rheumatoid arthritis patients., 17 (18), 1971-1978 https://doi.org/10.2217/pgs-2016-0125 (2016).

41. Sharma, S. et al. Purine biosynthetic pathway genes and methotrexate response in rheumatoid arthritis patients among north Indians. Pharmacogenet. Genomics, 19 (10), 823-828 https://doi.org/10.1097/fpc.0b013e328331b53e (2009).

42. Muralidharan, N., Mariaselvam, C. M., Jain, V. K., Gulati, R. \& Negi, V. S. ATIC 347C> G gene polymorphism may be associated with methotrexate-induced adverse events in south Indian Tamil rheumatoid arthritis. Pharmacogenomics 17(3), 241-248(2016). https://doi.org/10.2217/pgs.15.170

43. Lee, Y. H. \& Bae, S-C. Association of the ATIC 347 C/G polymorphism with responsiveness to and toxicity of methotrexate in rheumatoid arthritis: a meta-analysis. Rheumatol. Int, 36 (11), 1591-1599 https://doi.org/10.1007/s00296-016-3523-2 (2016).

44. Qiu, Q. et al. Polymorphisms and pharmacogenomics for the clinical efficacy of methotrexate in patients with rheumatoid arthritis: a systematic review and meta-analysis. Sci. Rep, 7 (1), 1-24 https://doi.org/10.1038/srep44015 (2017).

45. Dervieux, T. et al. Gene-gene interactions in folate and adenosine biosynthesis pathways affect methotrexate efficacy and tolerability in rheumatoid arthritis. Pharmacogenet. Genomics, 19 (12), 935-944 https://doi.org/10.1097/FPC.0b013e32833315d1 (2009).

46. Dervieux, T. et al. Patterns of interaction between genetic and nongenetic attributes and methotrexate efficacy in rheumatoid arthritis. Pharmacogenet. Genomics, 22, 1-9 https://doi.org/10.1097/FPC.0b013e32834d3e0b (2012).

47. Fales, K. R. et al. Discovery of N-(6-Fluoro-1-oxo-1, 2-dihydroisoquinolin-7-yl)-5-[(3 R)-3-hydroxypyrrolidin-1-yl] thiophene-2-sulfonamide (LSN 3213128), a potent and selective nonclassical antifolate aminoimidazole-4carboxamide ribonucleotide formyltransferase (AICARFT) inhibitor effective at tumor suppression in a cancer xenograft model. J. Med. Chem, 60 (23), 9599-9616 https://doi.org/10.1021/acs.jmedchem.7b01046 (2017).

48. Purcell, S. et al. PLINK: a tool set for whole-genome association and population-based linkage analyses. Am. J. Hum. Genet, 81 (3), 559-575 https://doi.org/10.1086/519795 (2007).

\section{Tables}

Table 1. Characteristics of the patients enrolled in this study. A total of 647 RA patients were stratified into three ethnic groups, Malay, Chinese and Indian. ${ }^{a}$ Data are presented in number (percentage) or mean (standard deviation) unless otherwise indicated; ${ }^{b}$ Rheumatoid factor; ${ }^{c}$ Anti-cyclic citrullinated peptide; ${ }^{d}$ Adequate responder; ${ }^{\mathrm{e}}$ Inadequate responder; ${ }^{\mathrm{f}}$ Non-adverse drug reaction; ${ }^{g}$ Adverse drug reaction. 


\begin{tabular}{|c|c|c|c|c|}
\hline Characteristics & Total $^{a}$ & Malay & Chinese & Indian \\
\hline Patients number, n (\%) & $647(100 \%)$ & $153(23.65 \%)$ & 326 (50.39\%) & $168(25.97 \%)$ \\
\hline \multicolumn{5}{|l|}{ Demographics } \\
\hline \multicolumn{5}{|l|}{-Gender } \\
\hline Female, n (\%) & $574(88.72 \%)$ & $131(85.62 \%)$ & $289(88.65 \%)$ & $154(91.67 \%)$ \\
\hline Male, n (\%) & $73(11.28 \%)$ & $22(14.38 \%)$ & $37(11.35 \%)$ & $14(8.33 \%)$ \\
\hline \multicolumn{5}{|l|}{-Age (years) } \\
\hline Mean (SD) & $56(12.10)$ & $53(11.63)$ & $58(12.02)$ & $56(11.95)$ \\
\hline Range & $18-92$ & $18-83$ & $21-92$ & $18-92$ \\
\hline \multicolumn{5}{|c|}{ Age of disease diagnosis (years) } \\
\hline Mean (SD) & $46(12.46)$ & 44 (12.39) & $48(12.51)$ & $46(12.15)$ \\
\hline Range & 9-89 & $11-80$ & $9-80$ & $16-89$ \\
\hline \multicolumn{5}{|l|}{-Disease duration (years) } \\
\hline Mean (SD) & $10(7.39)$ & $9(6.98)$ & $10(7.67)$ & $10(7.12)$ \\
\hline Range & $0.5-47$ & $1-36$ & $0.5-46$ & $1-47$ \\
\hline \multicolumn{5}{|l|}{ Clinical data } \\
\hline \multicolumn{5}{|l|}{$-\mathrm{RF}^{\mathrm{b}}$} \\
\hline RF positive RA, n (\%) & $525(81.14 \%)$ & $121(79.08 \%)$ & $262(80.37 \%)$ & $142(84.52 \%)$ \\
\hline RF negative RA, n (\%) & $121(18.70 \%)$ & $32(20.92 \%)$ & $63(19.33 \%)$ & $26(15.48 \%)$ \\
\hline \multicolumn{5}{|l|}{-Anti-CCPC } \\
\hline Anti-CCP positive RA, n (\%) & $490(75.73 \%)$ & $108(70.59 \%)$ & $259(79.45 \%)$ & $123(73.21 \%)$ \\
\hline Anti-CCP negative RA, n (\%) & $128(19.78 \%)$ & $43(28.10 \%)$ & $52(15.95 \%)$ & $33(19.64 \%)$ \\
\hline \multicolumn{5}{|l|}{-MTX efficacy, n (\%) } \\
\hline$A R^{d}$ & $252(41.79 \%)$ & $59(41.55 \%)$ & $129(42.16 \%)$ & $64(41.03 \%)$ \\
\hline $\mathbb{R}^{\mathrm{e}}$ & $352(58.21 \%)$ & $83(58.45 \%)$ & 177 (57.84\%) & $92(58.97 \%)$ \\
\hline \multicolumn{5}{|l|}{-MTX toxicity, n (\%) } \\
\hline Non-ADR ${ }^{f}$ & $448(69.24 \%)$ & 104 (67.97\%) & $236(72.39 \%)$ & 109 (64.88\%) \\
\hline$A D R^{g}$ & $199(30.76 \%)$ & $49(32.03 \%)$ & $90(27.61 \%)$ & $59(35.12 \%)$ \\
\hline
\end{tabular}


Table 2. Allele and genotype frequencies of the three ethnic groups in Malaysia. ${ }^{a}$ Data are presented in number (percentage) unless otherwise indicated. ${ }^{b}$ Data are presented in frequency (number) unless otherwise indicated;

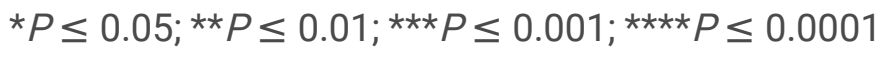




\begin{tabular}{|c|c|c|c|c|c|c|}
\hline SNP & & & Total & Malay & Chinese & Indian \\
\hline \multirow[t]{4}{*}{ FPGSA1994G (rs10106) } & \multirow[t]{3}{*}{$\begin{array}{l}\text { Genotype } \\
\text { Count }^{\mathrm{a}}\end{array}$} & AA & $111(17.16 \%)$ & $\begin{array}{l}21 \\
(13.73 \%)\end{array}$ & $\begin{array}{l}36 \\
(11.04 \%)\end{array}$ & $\begin{array}{l}54 \\
(32.14 \%)\end{array}$ \\
\hline & & AG & $283(43.74 \%)$ & $\begin{array}{l}74 \\
(48.37 \%)\end{array}$ & $\begin{array}{l}129 \\
(39.57 \%)\end{array}$ & $\begin{array}{l}80 \\
(47.62 \%)\end{array}$ \\
\hline & & GG & $253(39.10 \%)$ & $\begin{array}{l}58 \\
(37.91 \%)\end{array}$ & $\begin{array}{l}161 \\
(49.39 \%)\end{array}$ & $\begin{array}{l}34 \\
(20.24 \%)\end{array}$ \\
\hline & $\begin{array}{l}\text { Minor Allele } \\
\text { Frequency }\end{array}$ & & $0.61(789)$ & $\underset{\star \star \star \star \star}{0.62}(190)$ & $\underset{\star \star \star \star \star}{0.69}(451)$ & $\underset{\star \star \star \star \star \star}{0.44}(148)$ \\
\hline \multirow[t]{4}{*}{ GGHC452T (rs11545078) } & \multirow[t]{3}{*}{$\begin{array}{l}\text { Genotype } \\
\text { Count }^{\mathrm{a}}\end{array}$} & $\mathrm{CC}$ & $521(80.53 \%)$ & $\begin{array}{l}117 \\
(76.47 \%)\end{array}$ & $\begin{array}{l}276 \\
(84.66 \%)\end{array}$ & $\begin{array}{l}128 \\
(76.19 \%)\end{array}$ \\
\hline & & CT & $116(17.93 \%)$ & $\begin{array}{l}33 \\
(21.57 \%)\end{array}$ & $\begin{array}{l}48 \\
(14.72 \%)\end{array}$ & $\begin{array}{l}35 \\
(20.83 \%)\end{array}$ \\
\hline & & TT & $10(1.55 \%)$ & $3(1.96 \%)$ & $2(0.61 \%)$ & $5(2.98 \%)$ \\
\hline & $\begin{array}{l}\text { Minor Allele } \\
\text { Frequency }\end{array}$ & & $0.11(136)$ & $0.13(39)$ * & $0.08(52)$ * & $0.13(45)$ * \\
\hline \multirow[t]{4}{*}{ GGHC401T (rs3758149) } & \multirow[t]{3}{*}{$\begin{array}{l}\text { Genotype } \\
\text { Count }^{\mathrm{a}}\end{array}$} & $\mathrm{CC}$ & $356(55.02 \%)$ & $\begin{array}{l}69 \\
(45.10 \%)\end{array}$ & $\begin{array}{l}198 \\
(60.74 \%)\end{array}$ & $\begin{array}{l}89 \\
(52.98 \%)\end{array}$ \\
\hline & & CT & $237(36.63 \%)$ & $\begin{array}{l}63 \\
(41.18 \%)\end{array}$ & $\begin{array}{l}112 \\
(34.36 \%)\end{array}$ & $\begin{array}{l}62 \\
(36.90 \%)\end{array}$ \\
\hline & & TT & $54(8.35 \%)$ & $\begin{array}{l}21 \\
(13.73 \%)\end{array}$ & $16(4.91 \%)$ & $\begin{array}{l}17 \\
(10.12 \%)\end{array}$ \\
\hline & $\begin{array}{l}\text { Minor Allele } \\
\text { Frequency }\end{array}$ & & $0.27(345)$ & $\underset{\star \star \star \star}{0.34}(105)$ & $\underset{\star \star \star \star}{0.22}(144)$ & $\underset{\star \star \star \star}{0.29}(96)$ \\
\hline \multirow[t]{4}{*}{ ATIC C347G (rs2372536) } & \multirow[t]{3}{*}{$\begin{array}{l}\text { Genotype } \\
\text { Count }^{\mathrm{a}}\end{array}$} & $\mathrm{CC}$ & $239(36.94 \%)$ & $\begin{array}{l}44 \\
(28.76 \%)\end{array}$ & $\begin{array}{l}158 \\
(48.47 \%)\end{array}$ & $\begin{array}{l}37 \\
(22.02 \%)\end{array}$ \\
\hline & & CG & $292(45.13 \%)$ & $\begin{array}{l}80 \\
(52.29 \%)\end{array}$ & $\begin{array}{l}133 \\
(40.80 \%)\end{array}$ & $\begin{array}{l}79 \\
(47.02 \%)\end{array}$ \\
\hline & & GG & $116(17.93 \%)$ & $\begin{array}{l}29 \\
(18.95 \%)\end{array}$ & $\begin{array}{l}35 \\
(10.74 \%)\end{array}$ & $\begin{array}{l}52 \\
(30.95 \%)\end{array}$ \\
\hline & $\begin{array}{l}\text { Minor Allele } \\
\text { Frequency }^{\mathrm{b}}\end{array}$ & & $0.40(524)$ & $\underset{\star \star \star * \star *}{0.45}(138)$ & $\underset{\star \star \star * \star \star *}{0.31}(203)$ & 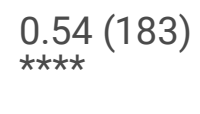 \\
\hline \multirow[t]{2}{*}{ ATIC T675C (rs4673993) } & \multirow[t]{2}{*}{$\begin{array}{l}\text { Genotype } \\
\text { Count }^{\mathrm{a}}\end{array}$} & TT & $241(37.25 \%)$ & $\begin{array}{l}46 \\
(30.07 \%)\end{array}$ & $\begin{array}{l}159 \\
(48.77 \%)\end{array}$ & $\begin{array}{l}36 \\
(21.43 \%)\end{array}$ \\
\hline & & $\mathrm{TC}$ & $296(45.75 \%)$ & $\begin{array}{l}80 \\
(52.29 \%)\end{array}$ & $\begin{array}{l}135 \\
(41.41 \%)\end{array}$ & $\begin{array}{l}81 \\
(48.21 \%)\end{array}$ \\
\hline
\end{tabular}




\begin{tabular}{|c|c|c|c|c|c|c|}
\hline & & $\mathrm{CC}$ & $110(17.00 \%)$ & $\begin{array}{l}27 \\
(17.65 \%)\end{array}$ & $32(9.82 \%)$ & $\begin{array}{l}51 \\
(30.36 \%)\end{array}$ \\
\hline & $\begin{array}{l}\text { Minor Allele } \\
\text { Frequency }^{\mathrm{b}}\end{array}$ & & $0.40(516)$ & $\underset{\star \star \star \star \star}{0.44}(134)$ & $\underset{\star \star \star * \star}{0.31}(199)$ & $\underset{\star \star \star 5 * \star}{0.54}(183)$ \\
\hline \multirow{4}{*}{$\begin{array}{l}\text { ITPA C94A } \\
\text { (rs1127354) }\end{array}$} & \multirow[t]{3}{*}{$\begin{array}{l}\text { Genotype } \\
\text { Count }^{a}\end{array}$} & CC & $460(71.10 \%)$ & $\begin{array}{l}110 \\
(71.90 \%)\end{array}$ & $\begin{array}{l}226 \\
(69.33 \%)\end{array}$ & $\begin{array}{l}124 \\
(73.81 \%)\end{array}$ \\
\hline & & CA & 177 (18.08\%) & $\begin{array}{l}40 \\
(26.14 \%)\end{array}$ & $\begin{array}{l}96 \\
(29.45 \%)\end{array}$ & $\begin{array}{l}41 \\
(24.40 \%)\end{array}$ \\
\hline & & AA & 10 (1.55\%) & $3(1.96 \%)$ & $4(1.23 \%)$ & $3(1.79 \%)$ \\
\hline & $\begin{array}{l}\text { Minor Allele } \\
\text { Frequency }^{b}\end{array}$ & & 0.15 (197) & $0.15(46)$ & $0.16(104)$ & $0.14(47)$ \\
\hline
\end{tabular}

Figures 


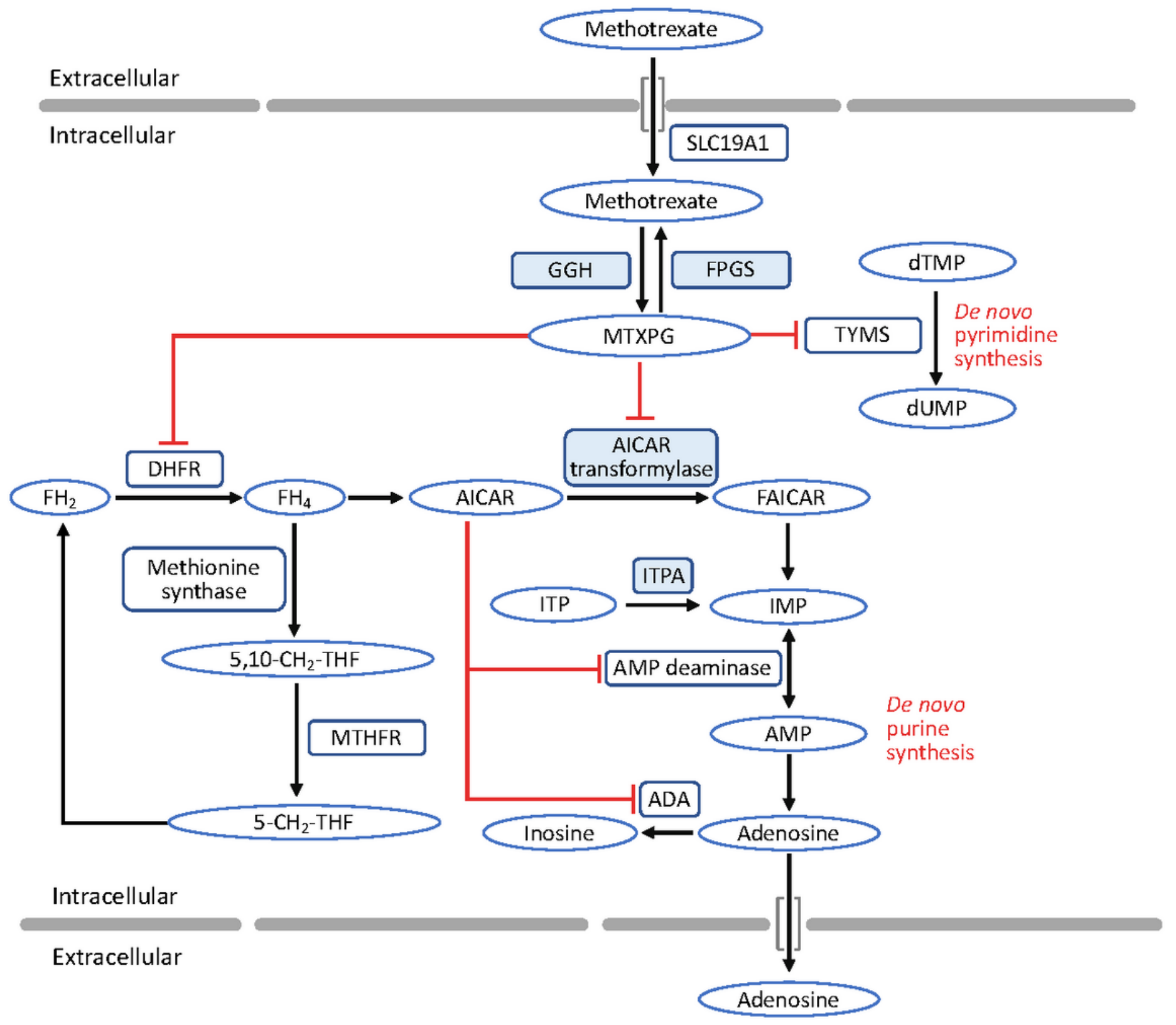

Figure 1

Cellular pathway of MTX - uptake, transport, conversion to polyglutamate forms and downstream effects. MTX is absorbed through active transport mediated by solute carrier family 19 member 1 (SLC19A1). Inside the cell, MTX is converted to active methotrexate polyglutamates (MTX PGs) by folylpolyglutamate synthase (FPGS) and this process can be reversed by y glutamyl hydro-lase (GGH). MTX PGs directly inhibit dihydrofolate reductase (DHFR), aminoimidazole-4-carboxamide ribonucleotide (AICAR) transformylase which is coded by ATIC and thymidylate synthetase (TYMS). Proteins highlighted in blue are encoded by the genes chosen for genotyping in this study. Red diamond $(\nabla)$ indicates the inhibitory activity and black arrowhead $(\boldsymbol{\nabla})$ indicates the directional flow of reaction. 

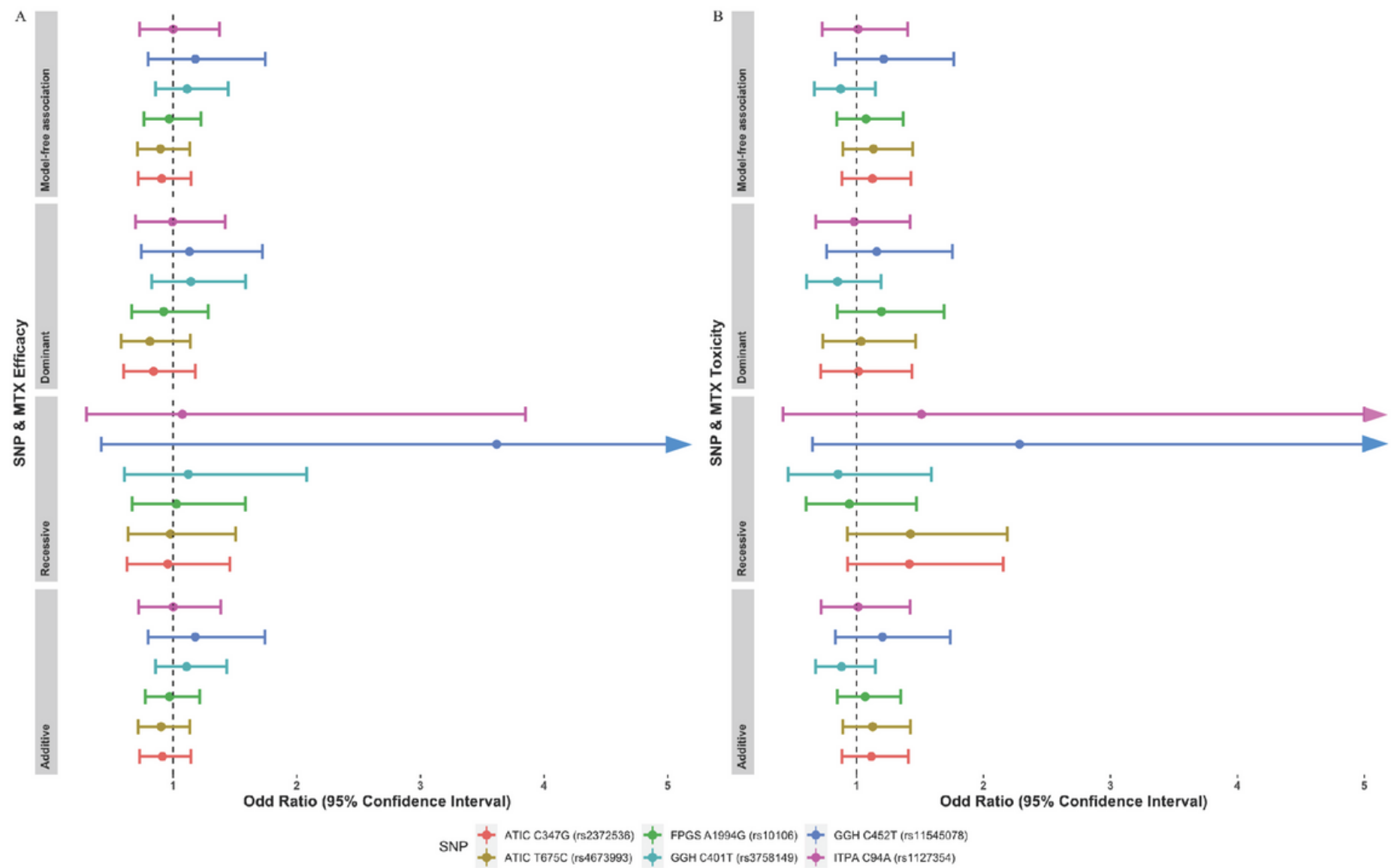

\section{Figure 2}

Forest plot showing the association between SNPs and either MTX efficacy or MTX Toxicity. A: Forest plot showing the association between SNPs and MTX efficacy; B: Forest plot showing the association between SNPS and MTX toxicity. A logarithmic scale was applied on the $\mathrm{x}$-axis. Circle points represent the OR of each test performed, and the results of $95 \% \mathrm{Cl}$ were displayed as a horizontal line. All the tests crossed the vertical line $(\mathrm{OR}=1.0)$, indicating that no significant association was found. 


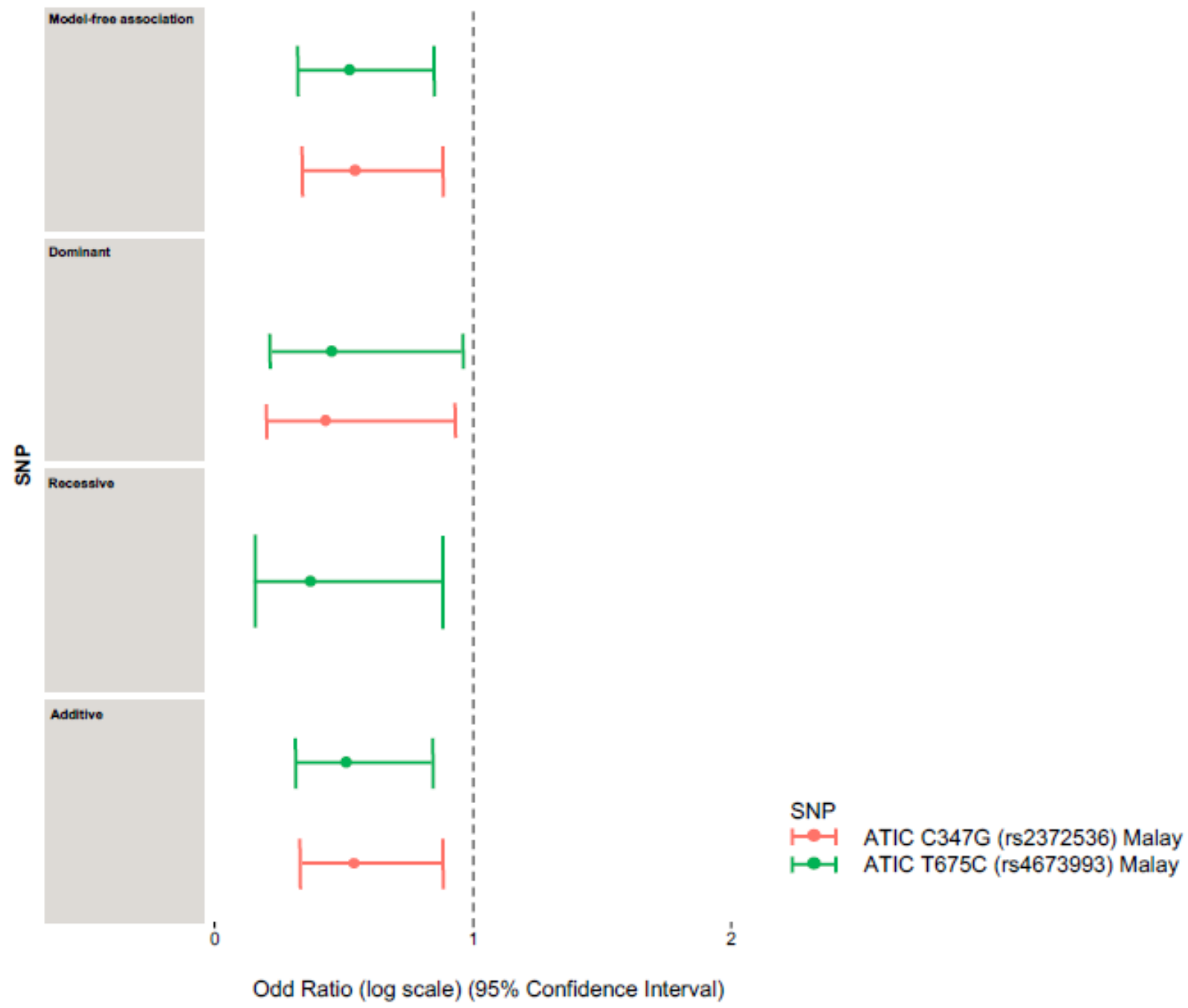

\section{Figure 3}

Forest plot showing a significant correlation of two ATIC SNPs with MTX efficacy in Malay RA patients. The forest plot was plotted by a logarithmic scale on the x-axis. The cycle dot represents the OR of each test performed, and the results of $95 \% \mathrm{Cl}$ were displayed as a horizontal line. 

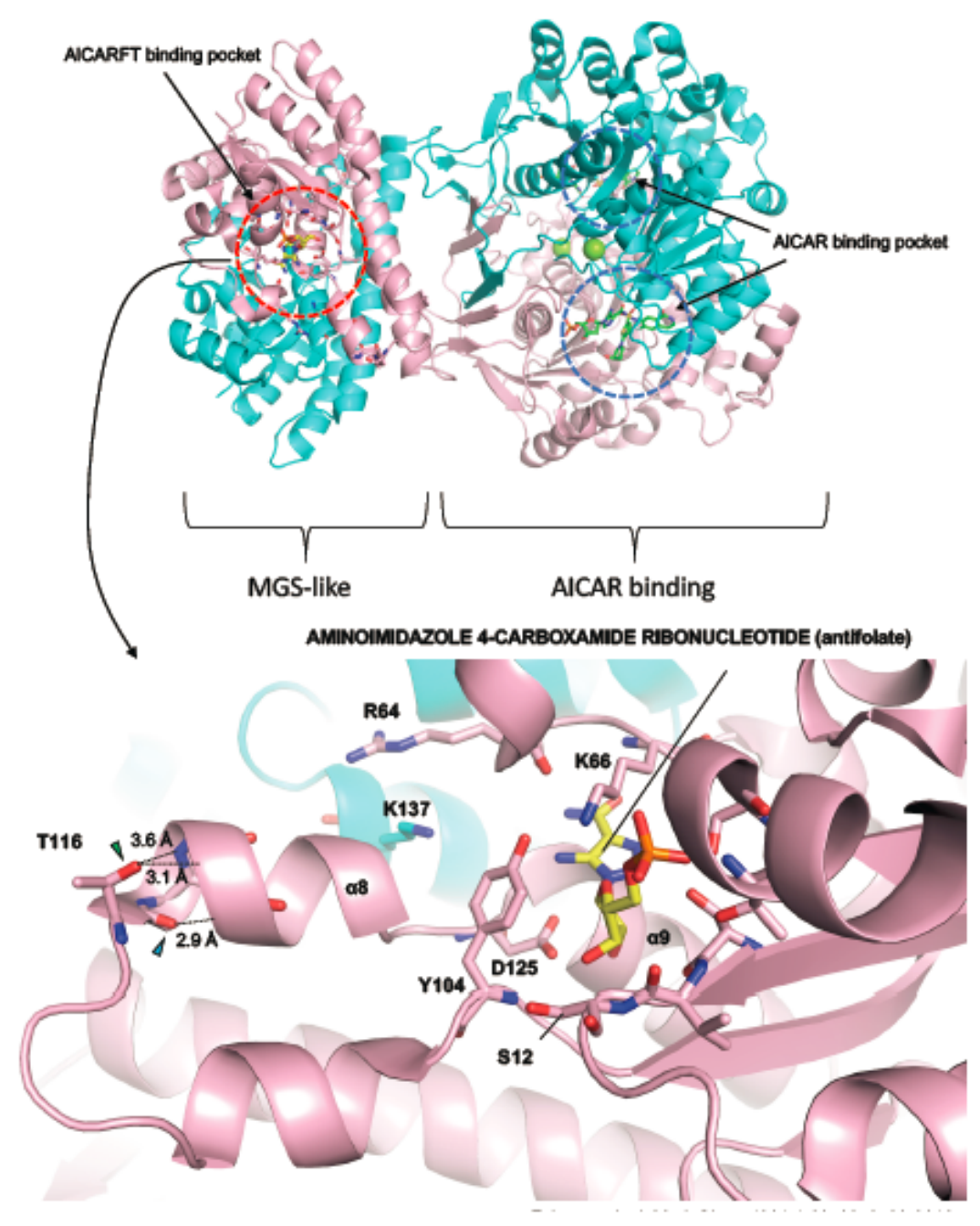

Figure 4

Tertiary structure of AICAR transformylase complexed with antifolate (aminoimidazole 4-carboxamide ribonucleotide) was retrieved from Protein Data Bank (https://www.rcsb.org) (PDB ID: 5UZ0)49. Thr116 (or T116) is in the MGS domain. The green arrowhead indicates the side-chain hydroxyl group of Thr116 that forms hydrogen bonds with the amide group from Val117 and Glu118. The blue arrowhead is where the main-chain carboxyl group of Thr116 forms hydrogen bond with the amide group of Glu119.

\section{Supplementary Files}

This is a list of supplementary files associated with this preprint. Click to download.

- SupplementaryTableS1S7Shaetal.docx 